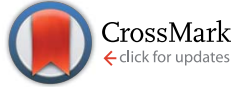

Cite this: RSC Adv., 2016, 6, 14832

Received 16th November 2015 Accepted 27th January 2016

DOI: $10.1039 / \mathrm{c} 5 \mathrm{ra} 24234 \mathrm{~g}$

www.rsc.org/advances

\section{Dual step irradiation process for in situ generation and patterning of silver nanoparticles in a photocured film $\uparrow$}

\author{
I. Roppolo, ${ }^{\star a}$ A. Doriguzzi Bozzo, ${ }^{b}$ M. Castellino, ${ }^{a}$ A. Chiappone, ${ }^{a}$ D. Perrone, ${ }^{a}$ \\ K. Bejtka, ${ }^{a}$ S. Bocchini, ${ }^{a}$ M. Sangermano ${ }^{b}$ and A. Chiolerio ${ }^{a}$
}

\begin{abstract}
The realization of nanocomposite materials with topologically tailored composition and functional properties represents an important technological challenge for many applications such as optics and electronics. In the last years a great effort has been devoted to develop techniques that, by controlling the generation and the distribution of nanoparticles, allow the realization of structured metal/polymer nanocomposites. In this work we present a novel methodology for the realization of patterned silver/ polymer nanocomposites generated in a polymeric matrix starting from a single formulation exploiting a fully photoinduced dual wavelength method, maintaining control over size distribution, with a straightforward influence on electronic/plasmonic/photonic properties. A step by step study of the process was carried out, investigating both the polymerization kinetics and the nanoparticles generation. Finally patterning in the polymeric matrix was performed through photolithographic technology and a washing process was developed in order to stabilize the material toward ageing. This technique opens new perspectives in the realization of multifunctional materials and devices exploiting electronic/ plasmonic/photonic properties of the embedded nanoparticles and it is envisaged to be further improved by applying region-selective techniques.
\end{abstract}

\section{Introduction}

Polymer-metal nanocomposites (NCs) are nowadays an important class of materials exploited in many different applications such as optics, ${ }^{1}$ photonics, ${ }^{2}$ electronics, ${ }^{3}$ electrical devices, ${ }^{4,5}$ bioactive materials ${ }^{6,7}$ and others. ${ }^{8-11}$

Among the various techniques developed to realize polymermetal NCs one of the most popular approaches consists of the in situ generation of metal nanoparticles (NPs) in a polymer matrix by embedding metal salts in the starting formulation as precursors. ${ }^{12}$ Various methodologies were developed for obtaining this type of NCs such as thermal, ${ }^{13-15}$ plasma induced $^{\mathbf{1 6}}$ and chemical reduction. ${ }^{\mathbf{1 7 , 1 8}}$ However, an interesting alternative method to obtain polymer NCs consists of the metal ions in situ photoreduction process. The photoinduced

${ }^{a}$ Istituto Italiano di Tecnologia, Center for Space Human Robotics, Corso Trento 21, 10129 Torino, Italy. E-mail: ignazio.roppolo@iit.it; Tel: +390115091907

${ }^{b}$ Politecnico di Torino, Applied Science and Technology Department, Corso Duca degli Abruzzi 24, 10129 Torino, Italy

$\dagger$ Electronic supplementary information (ESI) available: Picture of the artisanal device used for photopolymerization process; photorheology experiments; FT-IR spectra and analysis before and after curing; DSC thermograms; plasmon of resonance analysis, temperature measurement during the UV irradiation with and without mask; TGA measurements supplementary FESEM images; supplementary optical microscope pictures; picture of free-standing patterned film; pictures of aged samples. See DOI: 10.1039/c5ra24234g reduction of metal precursors has been a well established technique since the development of photography in the 19th century ${ }^{\mathbf{1 9}}$ and it has been deeply exploited in the latest 20 years for developing polymer NCs. ${ }^{20-22}$

More recently polymer-metal NC synthesis was carried out in a single step by means of photopolymerization processes. ${ }^{23-25}$ Photopolymerization is a fast, economic and energy saving technique allowing the realization of coating or free standing films by light irradiation, typically UV light. ${ }^{26}$ The UV irradiation of photocurable formulations containing metal salts of different elements such as gold and silver induces the simultaneous formation of a polymer network and the generation of metal NPs. ${ }^{27}$ The concurrent formation of inorganic and organic phase allows a good distribution of metal NPs in the polymeric network, avoiding agglomeration and limiting NPs size, thus realizing a polymer-metal $\mathrm{NC}$ in a very short time. ${ }^{21}$ Electrically conductive materials, ${ }^{28}$ capacitors $^{29}$ and materials suitable for electronic applications ${ }^{\mathbf{3 0}}$ were developed by this technique.

A further point investigated in the last few years consists of the control on the generation and distribution of NPs in the polymeric matrix by different techniques such as monochromatic UV-induced radiation, ${ }^{31-33}$ holographic exposure, ${ }^{34}$ laser radiation ${ }^{35,36}$ or SPM. ${ }^{37}$ By fully controlling the NPS generation it was thus possible to create materials featuring controlled distribution of NPs. These approaches could be useful for certain applications such as diffraction gratings or 
meta-materials. ${ }^{38,39}$ These techniques usually involve the swelling of polymeric materials in solutions containing the precursors of the metal phase in order to perform the infiltration in the polymer network. However, to the best of our knowledge, the proposed techniques still present some drawbacks: first of all the infiltration process implies a difficult control on the amount of the precursor released in the network and on its distribution in the polymeric matrix; moreover long exposure times are often requested (more than $24 \mathrm{~h}$ ) and 3dimensional control of the patterning still remains an issue. In our opinion, the main limit for all the reported approaches is the need of getting rid of the unreduced metal salts which will be detrimental for stability to ageing and for a standardization of the electronic properties of the produced materials.

In this frame the present work finds its reason. The goal consists of developing a full photoinduced process for the realization of patterned materials (selectively inhomogeneous from the compositional and functional point of view) starting from a homogeneous formulation. First a photocurable formulation containing photocurable monomers, suitable photoinitiators and metal salts (in our case silver nitrate) was irradiated in the visible range in order to realize a cross-linked polymer network. Visible induced polymerization is a less energetic alternative to UV-induced polymerization that involves the use of sensitizers that are able to generate initiating species (that could be either radicals or cations) which can carry out polymerization process, thus obtaining both coating and self standing materials. ${ }^{40-42}$ On the other side, since silver nitrate is not photoactive in the visible range, photoreduction of metal precursor was avoided, obtaining thus a photocured polymeric matrix still containing silver salts. The interest in silver comes from its properties: it is one of the most conductive metals available, it sustains plasmonic resonance and it does not suffer from fast oxidation, when in NPs form, like other metals as, for example, copper. ${ }^{43,44}$ The cured material was then patterned by UV-induced silver salts reduction through classical photolithographic method and finally a washing step in ethanol was performed in order to eliminate residual unreduced salts. In this way the salts were extracted from the cross-linked matrix maintaining the generated NPs in the polymer network. By following this process it was possible to realize easily metalpolymer NCs selectively inhomogeneous. The proposed process guarantees several advantages in comparison to other methods described in literature: first of all we feature a total control over the amount of metal dispersed in the polymeric matrix, by controlling the amount of precursor in the initial photocurable formulation, achieving also a homogeneous dispersion. Moreover the patterning process is performed on completely solid films, exploiting more standard photolithographic techniques; it is very flexible since it could be applied to any acrylic/ methacrylic monomer guaranteeing tailored final properties of the NCs. Finally the process, owing to its ease of implementation and speed, could be up-scaled for volume productions of patterned NC polymeric materials.

Step by step study of the process was carried out: first the visible light induced polymerization of the matrix was followed by means of photorheology and FT-IR analysis. Then the UV- induced generation process of Ag NPs was studied by UV-vis and FESEM, characterizing the size distribution as a function of irradiation time and evidencing a correlation between NPs' spatial distribution in the polymer matrix and irradiation time. Finally the patterning process was performed, exploiting photolithographic techniques in order to obtain structures resolved at the micron scale. The efficiency of the washing step was then evaluated by thermogravimetrical measurements and XPS.

The photopolymerization results unique for its versatility and allows an easy production of this new class of materials exploiting a two (or more) wavelength selection. In synthesis, by following the proposed method, we achieved a fast synthesis of novel NC materials with controlled structure, suitable for several electronic/plasmonic/photonic applications.

\section{Results and discussion}

The aim of the present work consists of developing a full lightcarried process for the realization of materials selectively inhomogeneous from the compositional point of view starting from a single photocurable formulation. The process that we propose is composed by three stages, sketched in Fig. 1: first the photocurable formulations were irradiated in the visible range in order to realize a cross-linked polymer network without reducing silver salts employing as photoinitiating system a mixture of camphorquinone (CQ) and ethyl 4 (dimethylamino) benzoate (EMBO); then the cured materials were patterned by UV-induced Ag nitrate reduction through classical photolithographic method using a UV photoinitiator (Irgacure 1173) as electron donor and finally a washing step in ethanol was performed. In this way it is possible to extract the unreduced ions from the cross-linked matrix without damaging the patterned structure. In order to fully control this process it is necessary

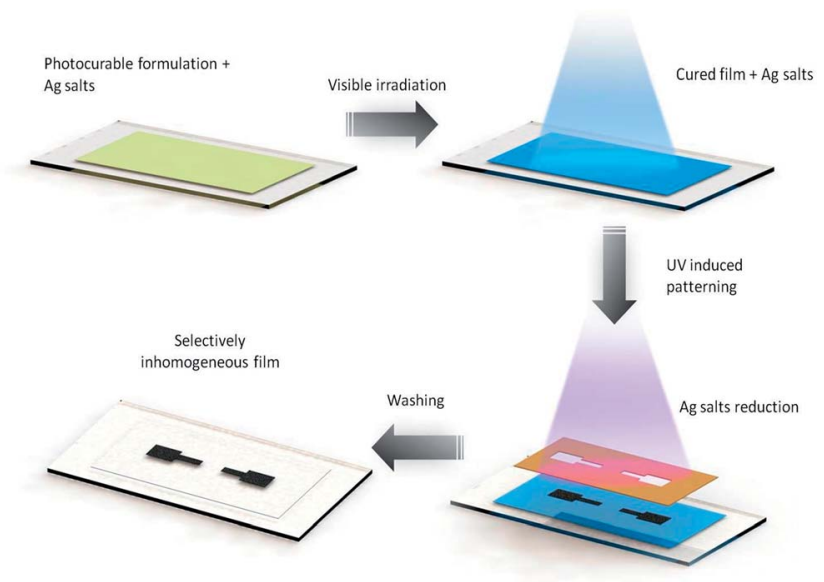

Fig. 1 Process layout for the realization of selectively patterned films by dual step irradiation process: in the first step a photocurable formulation containing silver nitrate is cross-linked by means of visible light irradiation, then patterned structures of Ag NPs are generated in the polymeric matrix by UV reduction exploiting photolithography; finally unreduced ions are extracted by means of ethanol washing. 
first to determine the kinetics of visible induced photopolymerization in order to obtain cured samples avoiding silver salt photoreduction.

\section{Visible induced photopolymerization}

The visible light photopolymerization kinetics was followed by means of photorheology which is known to be an effective method for characterizing the curing process of thermosetting polymers. Dynamic rheology has been applied in the past years to the characterization of the UV or visible photopolymerization of various systems. ${ }^{\mathbf{4 5 , 4 6}}$ The formation of polymer gels can be monitored from the time evolution of viscoelastic material functions such as $G^{\prime}$ and $G^{\prime \prime}$.

Fig. 2 a shows the evolution of the storage modulus $\left(G^{\prime}\right)$ during irradiation under visible light for the neat monomer (poly(ethyleneglycol) dimethacrylate, PEGDMA) and for the same monomer in the presence of different amounts of silver nitrate. The photopolymerization of the system is slightly influenced by the presence of $\mathrm{AgNO}_{3}$, in fact while for the photocurable formulation of neat PEGDMA and PEGDMA containing 1 phr of $\mathrm{Ag}$ salt the reaction instantaneously starts when the light is turned-on, and $G^{\prime}$ reaches its plateau in 3 minutes of irradiation, in the presence of 5 or $10 \mathrm{phr}$ of nitrate it is possible to observe a slowing down of the reaction.

In order to better understand the influence of the irradiation time on network formation, the gel point of the formulations has also been evaluated. Gel point gives an estimate of the time at which the resin begins to gel and it could be considered as the cross-over point of the two modulus curves (when $G^{\prime}>G^{\prime \prime}$ ). ${ }^{47}$ In Fig. 2b $G^{\prime}$ and $G^{\prime \prime}$ moduli of PEGDMA are reported as representative for all the cases. Despite the slight negative effect of the silver salt on the photopolymerization kinetics, the gel point resulted to occur within the first 20 seconds of irradiation for all the investigated formulations. It is important to minimize the irradiation time in order to avoid silver salt reduction (that could be induced both from visible light and from the heat arising from photopolymerization process ${ }^{25,34}$ ), so in accordance with the photorheology results, one minute of blue-light irradiation was selected as the time needed to obtain a film above its gel point. A quantitative evaluation of double bond conversion was followed by means of FT-IR analysis. The values of $\mathrm{C}=\mathrm{C}$ conversion are reported in Table 1 (full spectra are reported in $\mathrm{ESI}^{\dagger}$ ). As expected, for every sample the conversion was quite high (around 80\%) regardless of the amount of silver precursors in the formulation. This is a first indication that the radicals generated by the visible photoinitiating system do not undergo silver reduction but polymer network formation. Also, such high values mean that in one minute of irradiation an almost fully-cured, tacky-free sample could be obtained. All the cured samples were then irradiated for a further minute under the UV light in order to investigate the progress of the polymerization under a more energetic light source. The data reported in Table 1 show a moderate increase of conversion, indicating that visible radiation was sufficient to obtain a solid sample, even if less converted. This is confirmed by evaluation of gel content after $24 \mathrm{~h}$ chloroform abstraction: the sample of PEGDMA cured under visible light shows a very high insoluble fraction (92\%) very close to that of the UV-irradiated sample. Moreover the samples containing reduced silver salts show the same conversion and the same gel content of the neat PEGDMA after UV exposure, indicating that the presence of silver NPs does not affect the curing process. So in conclusion, the presence of silver salt could slightly affect the photopolymerization rate without significantly affecting the final double bond conversion.

The small difference in polymerization degree is also confirmed by DSC measurements (see Table 1, full thermograms
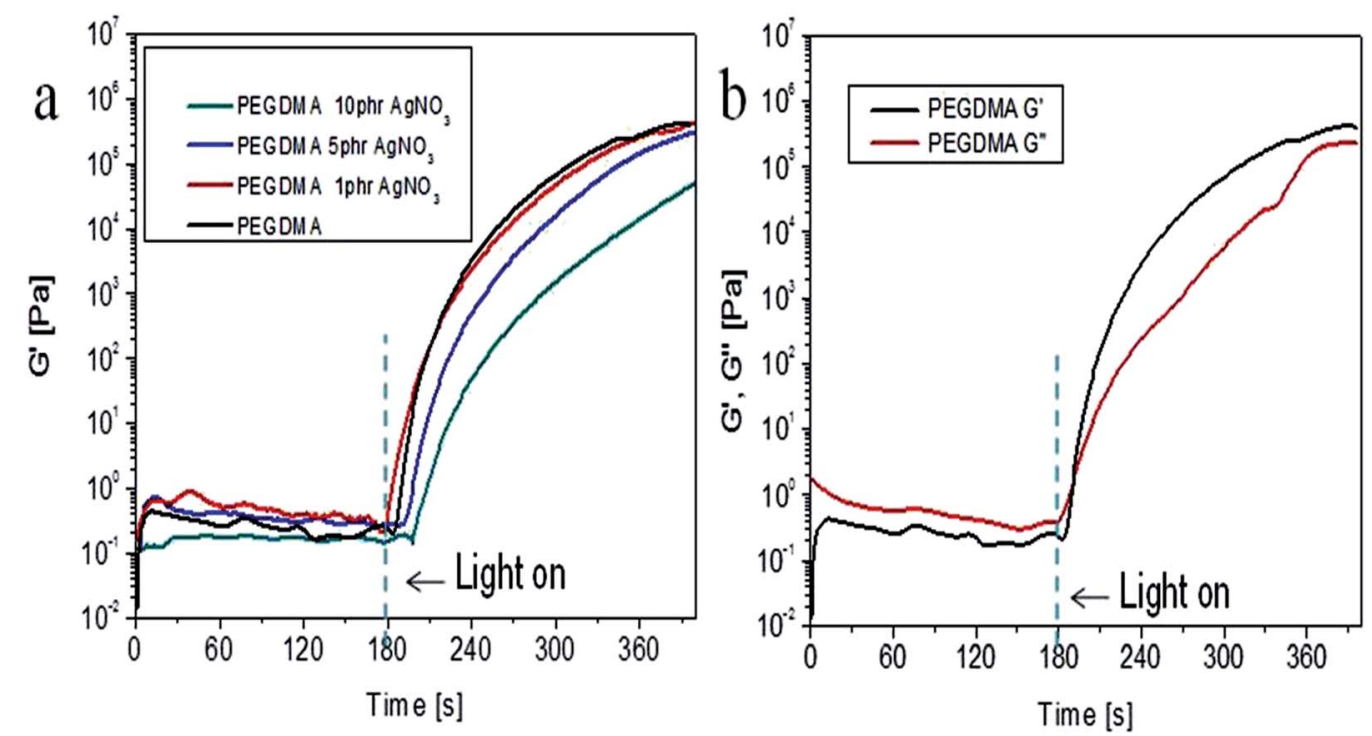

Fig. 2 (a) Time dependence of $G^{\prime}$ at $25^{\circ} \mathrm{C}$ and $1 \mathrm{rad} \mathrm{s}^{-1}$ for the visible light polymerization process of PEGDMA in the presence of different quantities of silver nitrate. Dotted line indicates the turning on of the lamp after 3 minutes from the beginning of the measurement. (b) Time dependence of $G^{\prime}$ and $G^{\prime \prime}$ for sample PEGDMA reported as example, the gel point is obtained from intersection point of $G^{\prime}$ and $G^{\prime \prime}$. 
Table 1 Conversion data, insoluble fraction and $T_{\mathrm{g}}$ of the sample tested

\begin{tabular}{|c|c|c|c|c|}
\hline Sample & $\begin{array}{l}\text { Conversion (\%) } \\
\text { after visible } \\
\text { irradiation }\end{array}$ & $\begin{array}{l}\text { Conversion (\%) } \\
\text { after further } \\
\text { UV irradiation }\end{array}$ & $\begin{array}{l}\text { Gel } \\
\text { content (\%) }\end{array}$ & $\begin{array}{l}T_{\mathrm{g}} \\
\left({ }^{\circ} \mathrm{C}\right)\end{array}$ \\
\hline PEGDMA & 81 & & 92 & -22 \\
\hline $\begin{array}{l}\text { PEGDMA } \\
\text { (after UV } \\
\text { irradiation) }\end{array}$ & - & 88 & 95 & -15 \\
\hline $\begin{array}{l}\text { PEGDMA + } \\
1 \text { phr AgNO }\end{array}$ & 85 & 93 & 94 & -15 \\
\hline $\begin{array}{l}\text { PEGDMA + } \\
5 \text { phr } \mathrm{AgNO}_{3}\end{array}$ & 87 & 95 & 95 & -12 \\
\hline $\begin{array}{l}\text { PEGDMA + } \\
10 \text { phr AgNO }\end{array}$ & 76 & 89 & 93 & -10 \\
\hline
\end{tabular}

in ESI $\dagger$ ). In fact, PEGDMA after visible curing shows a $T_{\mathrm{g}}$ of $-22{ }^{\circ} \mathrm{C}$ while after UV irradiation it increases up to $-15^{\circ} \mathrm{C}$. This is due to the increase of cross-linking density evidenced also by FT-IR measurements. In the sample containing Ag NPs after 1 min UV irradiation a small increase of $T_{\mathrm{g}}$ by increasing the silver content was measured, indicating that the presence of silver NPs generated in the polymer matrix slightly hinders the chain motion, leading thus to an increase of $T_{\mathrm{g}}$. In comparison to what reported for systems containing protic macromolecules such as glycogen ${ }^{48}$ or polyacrylic acid, ${ }^{49}$ the interaction between silver NPs is less strong and does not alter the infrared spectrum of cured PEGMA (see ESI $\dagger$ ).

\section{UV-induced silver NPs formation}

The subsequent step consisted in following the kinetics of silver NPs formation under UV irradiation. As known since the $19^{\text {th }}$ century, under UV-irradiation and in presence of an electron donor compound, silver ions undergo the reduction from $\mathrm{Ag}^{+}$to $\mathrm{Ag}^{0}{ }^{0}{ }^{19}$ Then metallic atoms tend to agglomerate forming $\mathrm{Ag}$ NPs embedded in the polymeric matrix. ${ }^{50}$ In the present work NP generation was carried out by UV exposure of cured samples containing unreduced silver salts employing Irgacure 1173 as electron donor activated by UV irradiation.

UV-visible spectra of the starting PEGDMA formulation containing CQ are reported in Fig. 3a and compared with the UV-vis spectra of the formulation after visible curing. As a further comparison the UV-vis spectrum of PEGDMA containing 5 phr of silver nitrate after visible curing is reported.

It is possible to observe that during the polymerization process the large peak centered around $480 \mathrm{~nm}$ attributed to CQ disappeared, indicating the effectiveness of visible induced mechanism. The two curves of the visible polymerized films are almost overlapped, indicating that no silver reduction occurred during visible irradiation (otherwise the typical silver plasmon resonance peak around $450 \mathrm{~nm}$ would have been detectable ${ }^{27}$ ). On the contrary the UV photoinitiator (Irgacure 1173) is not degraded as demonstrated by the presence of a shoulder peak around $320 \mathrm{~nm}$ in both spectra of the crosslinked films. The sample containing $5 \mathrm{phr}$ of $\mathrm{AgNO}_{3}$ was then irradiated with UVlight for different times in order to follow the silver NPs formation, full spectra are reported in Fig. 3b. By observing absorbance in log scale (Fig. 3c) it is possible to note
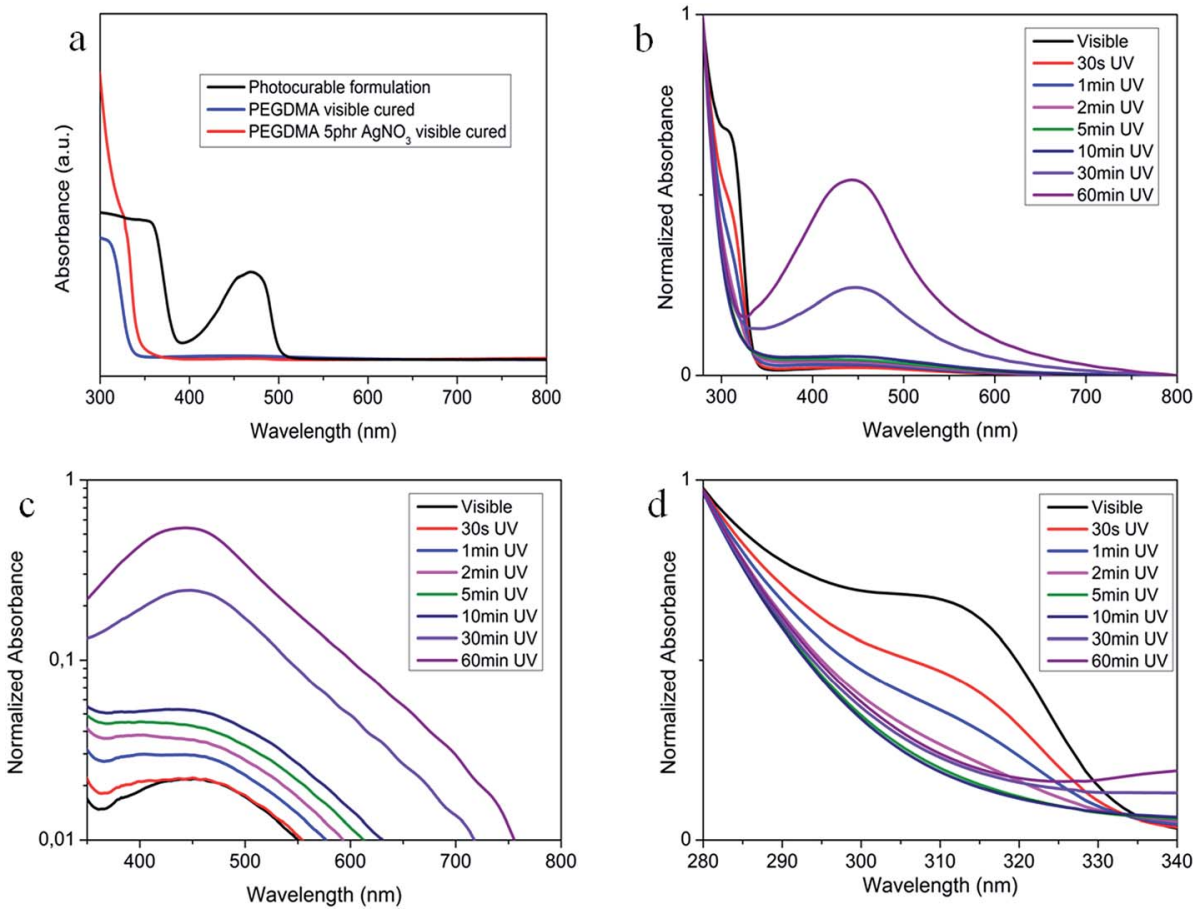

Fig. 3 UV-visible spectra: (a) comparison of uncured PEGDMA formulation, PEGDMA and PEGDMA + 5 phr AgNO 3 after visible-light induced curing; (b) growth kinetics of Ag NPs under UV-light at different irradiation times; (c) log-scale graph of the visible part of graph (b); (d) UVcomponent of graph (b). 
a progressive increase of the plasmon of resonance with the increase of the irradiation time, indicating that the metallic $\mathrm{Ag}$ organizes in NPs, formed through a nucleation/growth process.

However, a very evident peak is present only for long irradiation times (30 $\mathrm{min}^{-1}$ hour) while for low irradiation times the curves are almost overlapped. Since the Surface Plasmon Resonance (SPR) phenomenon is related to the dimensions and distances between silver NPs ${ }^{51,52}$ this means that longer times are necessary to obtain a system in which silver NPs are numerous and packed closely enough to obtain a large SPR peak. Since silver NPs nucleation and growth here occurs in a solid medium it is possible to foresee that longer times are necessary to obtain a large number of silver NPs. A more detailed analysis of the plasmon of resonance is reported in ESI. $\dagger$

Finally, by observing the UV part of the spectra (Fig. 3d) it is possible to note that the photoinitiator is fully consumed in the first two minutes of UV irradiation. The radicals generated in the homolytic cleavage of the UV-photoinitiator contribute to reduce the silver salts in the first stages of the reaction ${ }^{20}$ but in our opinion, this decrease is not directly correlated to the high increase of the peak centered around $450 \mathrm{~nm}$, whose origin should be related to other causes and will be discussed later.

Macroscopically, the formation of Ag NPs has an optically visible effect on the film. In Fig. 4 optical images of the same film after different irradiation times are showed. The visible cured film is transparent, indicating that no silver reduction occurred during polymerization step (Fig. 4a). Under UV irradiation silver NP formation occurs, changing the film color from yellow to brown (Fig. 4b-d) reaching finally a silver-mirrorlike appearance (Fig. 4e and f). This is in good agreement with the UV-visible measurements: in the first stage of the UV irradiation the samples maintain a similar color while for longer times a significant color change occurred. This color effect is related to the NPs size and distribution in the polymeric matrix. Fig. 5 shows the top view FESEM images of samples containing 5 phr of $\mathrm{AgNO}_{3}$ under different UV irradiation time (Fig. 5a-f) and numerical analysis of the imaged particles showing statistics on their equivalent diameter, distance, eccentricity and size distribution histograms (Fig. $5 \mathrm{~g}$ and h). ${ }^{53}$ The number of NPs and their maximum size increase as a function of the irradiation time. The average size is nearly constant, as may be understood considering the numerical estimates given in panel (Fig. 5g) (with huge standard deviation) and the histogram distribution given in panel (Fig. 5h). Interestingly the size distribution of sample exposed $10 \mathrm{~min}$ (blue) gives a clear proof of the nucleation and growth process, featuring a bimodal distribution with a first peak centered at 1.5 $\mathrm{nm}$ (nuclei) and a second peak centered at $9 \mathrm{~nm}$ (growing particles). For longer expositions, the first peak is covered by growing population (30 min, green) until much bigger particles appear ( $1 \mathrm{~h}$, orange). The distance between particles drops from 15 to around $5 \mathrm{~nm}$ in the sample irradiated at longer times (Fig. 5g), leading to mirror-like appearance and surface plasmon resonance phenomena. The eccentricity (spherical particles have 1 , ideally infinite aspect ratio nanotubes have 0 ) is always above 0.7 and their seems to be no correlation with the exposure time. The cross section FESEM images of these samples are shown in the Fig. 6, confirming a change in NPs number and distribution through the polymer matrix (see also Fig. S25-S27 in ESI†).

For low irradiation time fewer NPs are present and the most of them are localized at the NCs surface. This could be explained considering that during UV-irradiation the NPs
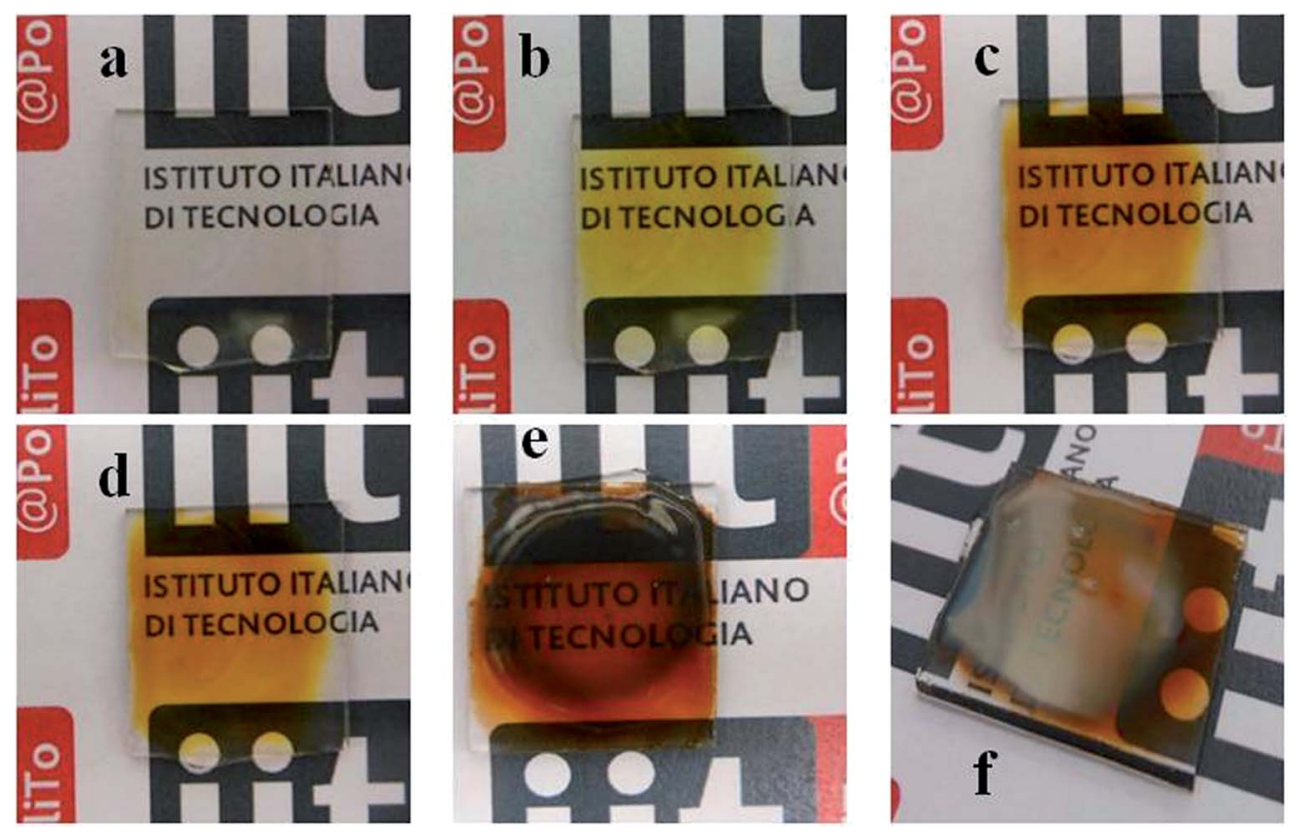

Fig. 4 Images of PEGDMA containing 5 phr $\mathrm{AgNO}_{3}$ (a) after visible-light induced polymerization; (b)-(e) after different UV irradiation time (1 min, 10 min, 30 min and 60 min respectively); (f) reflected light view of PEGDMA + 5 phr $\mathrm{AgNO}_{3}$ after 60 minutes UV irradiation. 

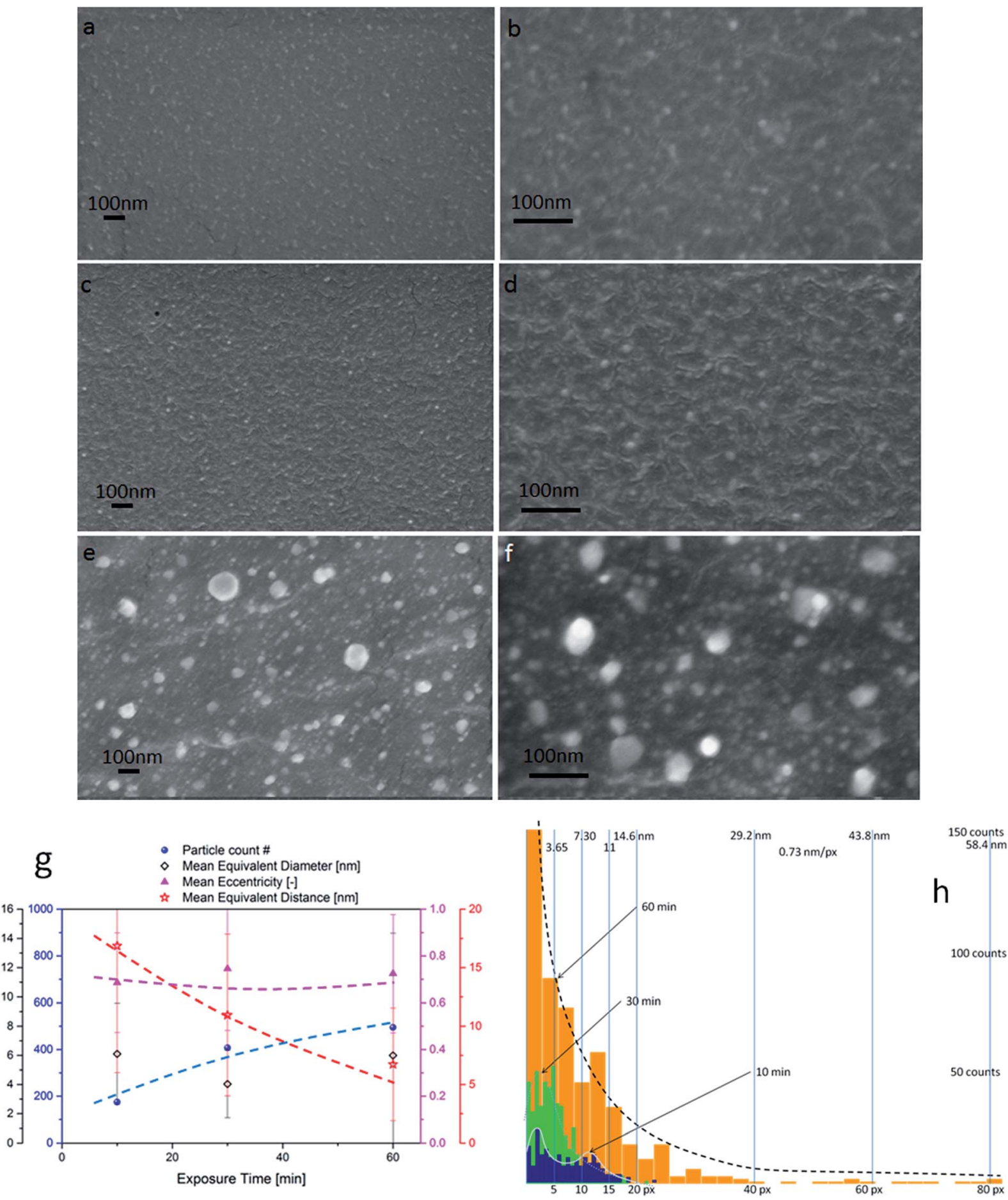

Fig. 5 (Top view) FESEM images PEGDMA containing 5 phr AgNO 3 after: (a and b) 10, (c and d) 30 and (e and f) 60 min of UV irradiation. The images in the right column (b, $d$ and f) are higher magnification of those in the left column ( $a, c$ and e). (g) Numerical analysis of the same images; (h) size distribution histograms. Lines are providing the trend and do not represent any fit.

nucleate and grow close to the light source since initial photoexcitation of silver ions occurs there. Increasing irradiation time results in a more homogeneous distribution of silver NPs throughout all polymer matrix indicating that with progressing the irradiation more silver salts were reduced. In the sample submitted to $1 \mathrm{~h}$ irradiation all NPs accumulated close to the surface while the rest of the matrix is almost neat.
A similar phenomenon was already observed in literature ${ }^{31,32}$ but to the best of our knowledge it is still unexplained. Since the already formed NPs have a diameter of tens of nm or more, we do not see that a migration through the matrix could take place without destroying the cross-linked network. On the other hand, Matsubayashi and coworkers showed that during the irradiation the NPs are subjected to a dissolution-reconstruction 

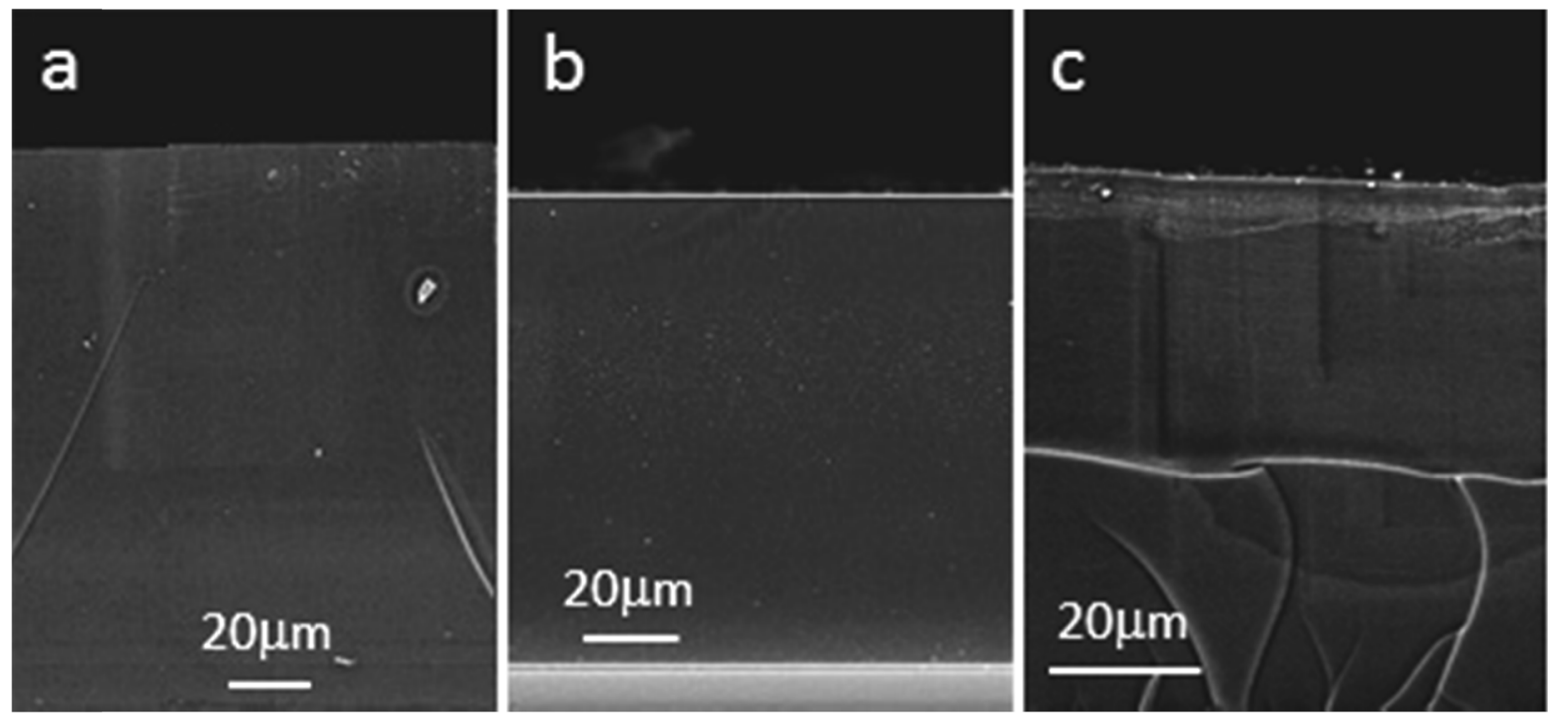

Fig. 6 Cross-section FESEM images of PEGDMA containing 5 phr $\mathrm{AgNO}_{3}$ after: (a) 10, (b) 30 and (c) 60 min of UV irradiation.

mechanism, generating small silver clusters with considerably lower dimensions and higher mobility. ${ }^{31}$ Based on this work, we propose that the silver-mirror-like appearance is governed by heat gradient in the polymeric matrix. This is in good agreement with other works in which thermally generated Ag NPs showed similar surface accumulation. ${ }^{\mathbf{1 3}, 15}$ In the present case we measured the temperature increase in the polymeric film during the UV irradiation (see ESI $\dagger$ ) by using a thermocouple placed in the polymeric film but not under direct UV light, to avoid heat transfer through irradiation. A temperature increase of $16^{\circ} \mathrm{C}$ was measured in one hour. For following experiments (see patterning section), a similar polymeric film was irradiated with a photomask maintaining the same light intensity on the film surface while monitoring the temperature in the film. In this case the temperature increase measured was lower $\left(12{ }^{\circ} \mathrm{C}\right)$ and all the produced materials showed a dark brown color without reaching the mirror-like appearance. Another possible reason for the temperature increase on the surface could be polymer photooxidation (see XPS measurements). We propose that during the irradiation a temperature gradient occurs, with a maximum temperature reached on the directly irradiated surface, and thus silver clusters generated during the irradiation are forced to accumulate on the film surface due to classical diffusivity laws.

\section{Washing step and patterning}

The last step of the proposed procedure consists of the patterning and removing of unreduced salts. In order to perform and efficient and stable patterning, first the silver salt removal was established. Ethanol was chosen as extracting solvent since water swelled the polymer matrix too much, causing undesired cracks.

Visible cured samples containing unreduced silver nitrate were then placed in ethanol for different times, to optimize the process salts. The silver residual was then evaluated by TGA experiments (see ESI $\dagger$ ) after $4 \mathrm{~h}$ in ethanol. Data shown in
Table 2 reports the residue at $800{ }^{\circ} \mathrm{C}$ after TGA measurements for unwashed and washed films. As expected, for all the unwashed samples the real residue is very close to the theoretical one. The efficiency of washing step was demonstrated since no residues were found in the samples containing silver nitrate after this treatment. In order to have a further confirmation, half of a cured sample was exposed to UV irradiation for 30 minutes, causing the generation of NPs evidenced by the coloring of the film. Then the whole film was washed for 4 hours in ethanol and then exposed for others 30 minutes to UV light. As a result the unexposed half remained transparent since all the silver nitrate was extracted (see Fig. 7).

A film containing 5 phr of $\mathrm{AgNO}_{3}$ was then analyzed by XPS in order to check the efficiency of the removal step and to obtain more information about the generation of silver NPs. In Fig. 8a the survey spectra of patterned (black line) and washed (red line) samples are reported. A strong reduction in the Ag intensity peaks is clearly visible on the un-patterned sample after the

Table 2 Residues measured after TGA experiments

\begin{tabular}{|c|c|c|c|}
\hline Sample & $\begin{array}{l}\text { Theoretical } \\
\text { residue }^{a}(\%)\end{array}$ & $\begin{array}{l}\text { Residue @ } \\
800{ }^{\circ} \mathrm{C}(\%)\end{array}$ & $\begin{array}{l}\text { Residue @ } \\
800{ }^{\circ} \mathrm{C}(\%) \\
\text { after washing step }\end{array}$ \\
\hline PEGDMA & 0 & 0 & - \\
\hline $\begin{array}{l}\text { PEGDMA } \\
\text { (after UV } \\
\text { irradiation) }\end{array}$ & 0 & 0 & - \\
\hline $\begin{array}{l}\text { PEGDMA + } \\
1 \text { phr } \mathrm{AgNO}_{3}\end{array}$ & 0.6 & 0.9 & 0 \\
\hline $\begin{array}{l}\text { PEGDMA + } \\
5 \text { phr } \mathrm{AgNO}_{3}\end{array}$ & 3.0 & 2.7 & 0 \\
\hline PEGDMA + & 5.6 & 4.9 & 0 \\
\hline
\end{tabular}
$10 \mathrm{phr} \mathrm{AgNO}_{3}$

${ }^{a}$ Calculated as theoretical content of silver in the samples. 


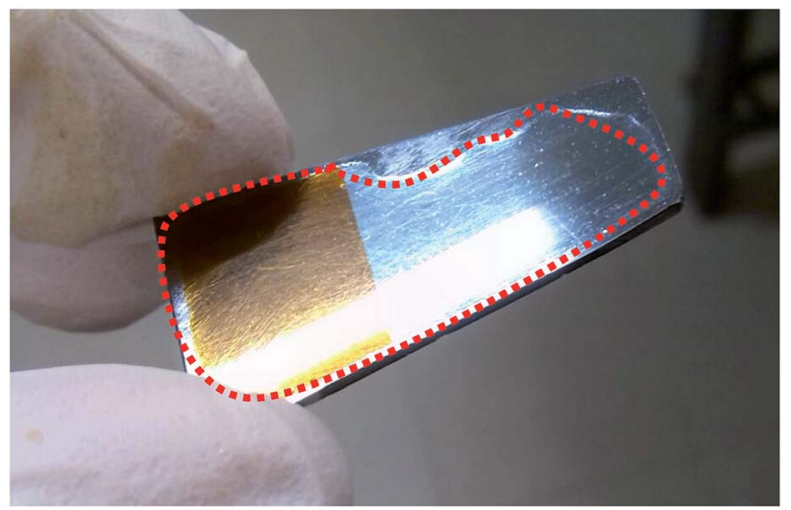

Fig. 7 Picture of PEGDMA + 5 phr $\mathrm{AgNO}_{3}$. After visible induced curing, one half was UV irradiated for 30 minutes, then the whole film was washed for 4 hours and subsequently irradiated for other 30 minutes under UV light. The right half remained transparent, indicating the efficiency of the washing step. washing procedure ( $\mathrm{Ag}$ is $<0.5$ at $\%$, while it is 1.7 at $\%$ on the patterned sample). It has to be understood that XPS technique is really sensitive to surface contamination, so even if the $\mathrm{Ag}$ is not detected with other bulk techniques, in this case we are able to detect also contaminations. In Fig. $8 \mathrm{~b}$ the Ag3d HR spectrum is reported together with its deconvolution curves, obtained by means of a mixed Gauss-Lorentz curve fitting. Reference value of binding energy for metallic $\mathrm{Ag}$ is $368.3 \mathrm{eV} .{ }^{54}$ In our case, however, the position of $\mathrm{Ag} 3 \mathrm{~d}_{5 / 2}$ peak is shifted towards lower binding energies $(368.0 \mathrm{eV})$. This peak position is quite close also to the binding energy of $367.8 \mathrm{eV}$, which corresponds to $\mathrm{Ag}_{2} \mathrm{O}$. Based on the surface sensitivity of XPS, it could be assumed that the detected peak corresponds to a layer of $\mathrm{Ag}_{2} \mathrm{O}$ formed on the surface of the Ag NPs. As already reported by Milošević et al. ${ }^{55}$ the attenuation length in silver NPs is such that the signal arises only from the top $4 \mathrm{~nm}$ of the surface, with the majority of the signal belonging to the outermost $1.5 \mathrm{~nm}$.
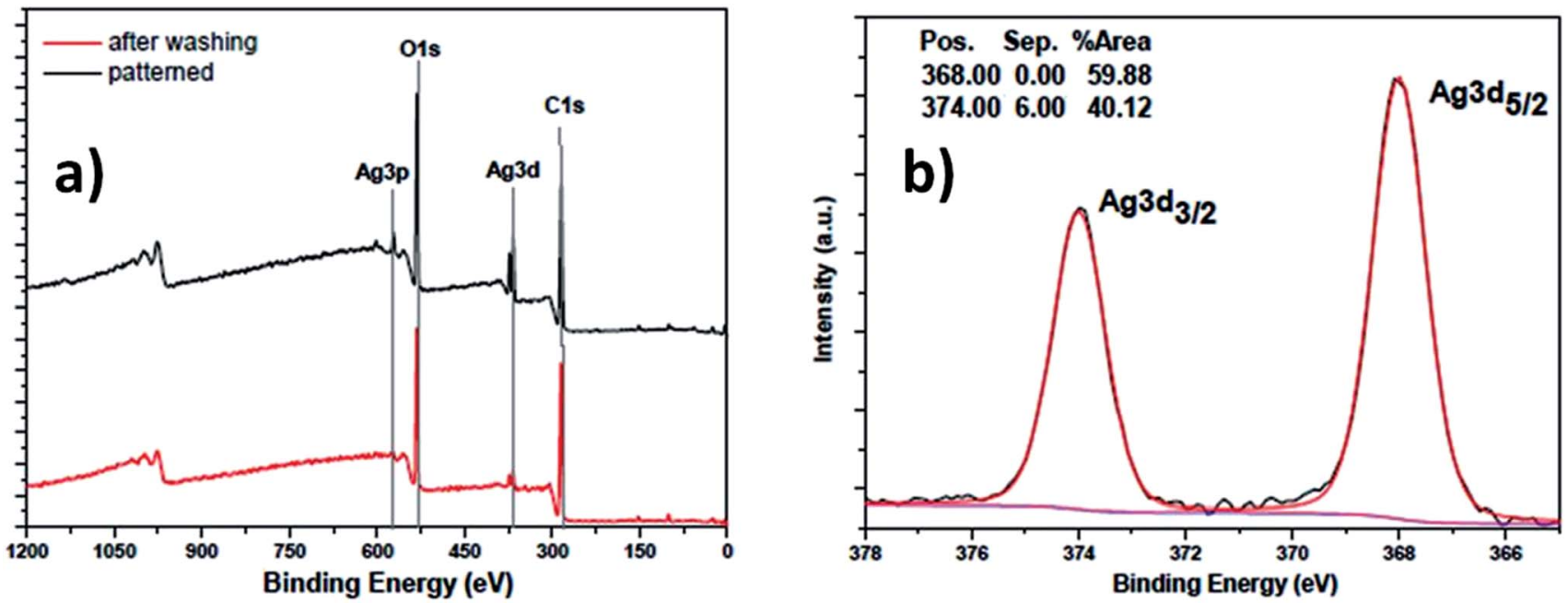

Fig. 8 (a) XPS survey spectra of patterned sample (black line) and un-patterned region after washing procedure (red line). (b) Ag3d doublet HR spectrum of patterned sample with deconvolution parameters obtained after Shirley background subtraction and mixed Gauss-Lorentz curve fitting.
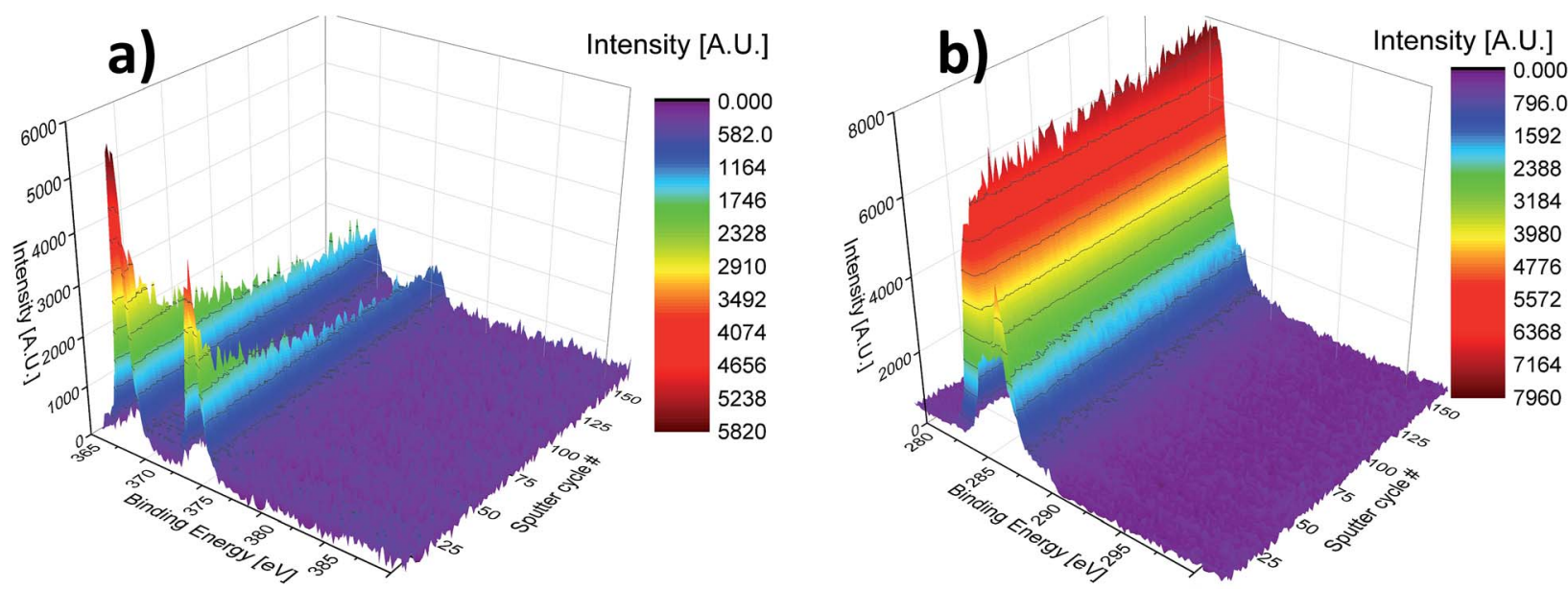

Fig. 9 (a) XPS Ag3d doublet and (b) C1s HR spectra of patterned sample during depth profile analysis (160 cycles). 


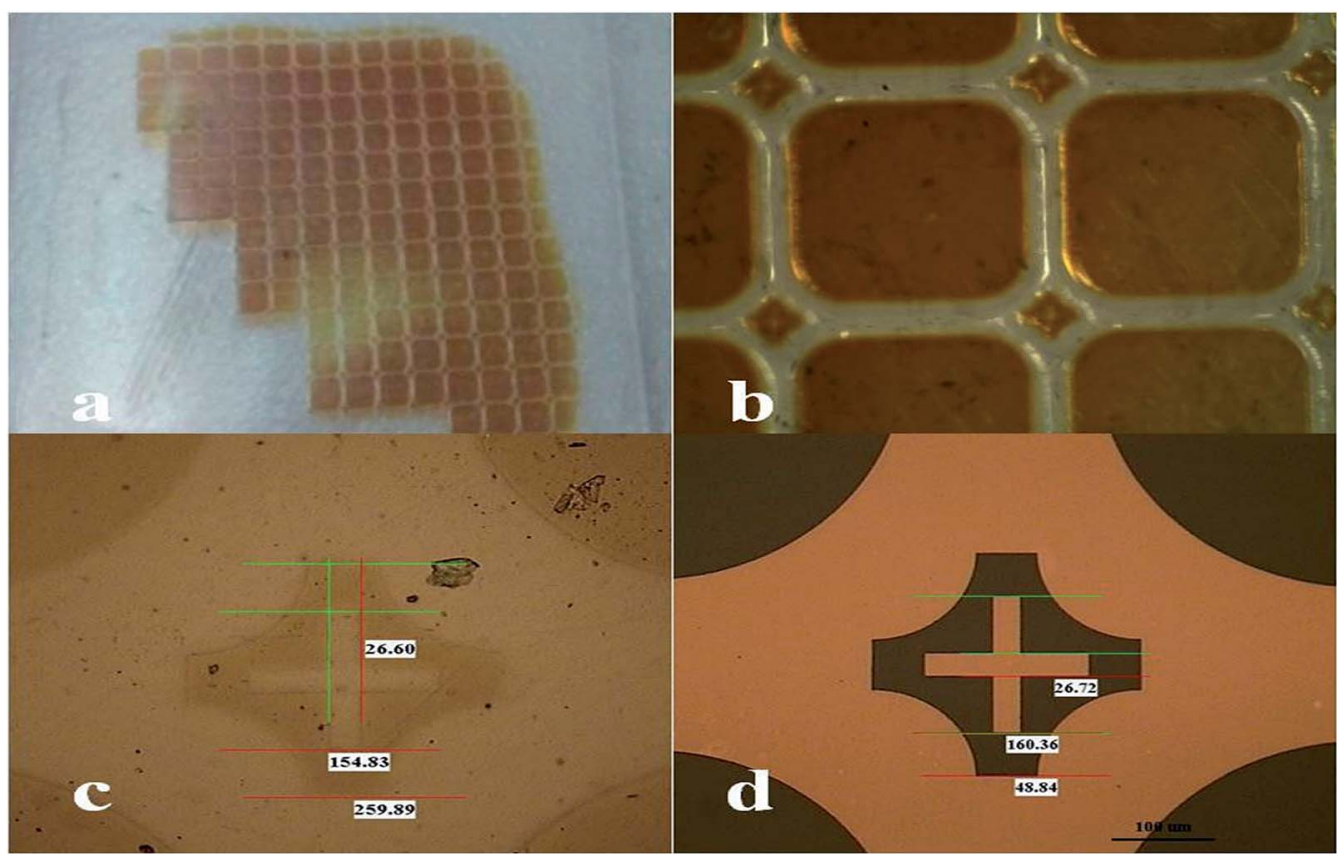

Fig. 10 Picture of patterned PEGDMA + 5 phr $\mathrm{AgNO}_{3}$ after washing step (a) full film (b) detail (c) optical microscopy (100 $\times$ ) of the patterned structure (d) optical picture of the mask used for the patterning.

Considering the dimensions of our NPs, this means that we are detecting only the first few oxide atomic layers. In order to assess this issue we have performed $\mathrm{Ar}^{+}$depth profiling, also to confirm the surface accumulation of silver NPs in depth. In Fig. 9a the Ag HR spectra produced during 160 cycles of depth profiling are reported. The intensity peak reduction is clearly visible starting from the $20^{\text {th }}$ cycle, confirming the accumulation in the first layers of the sample, while a shift towards higher binding energies $\left(368.2 \mathrm{eV}\right.$ ) has been noted for the $\mathrm{Ag} 3 \mathrm{~d}_{5 / 2}$ peak as expected. The shift is due to the removal of the surface oxide
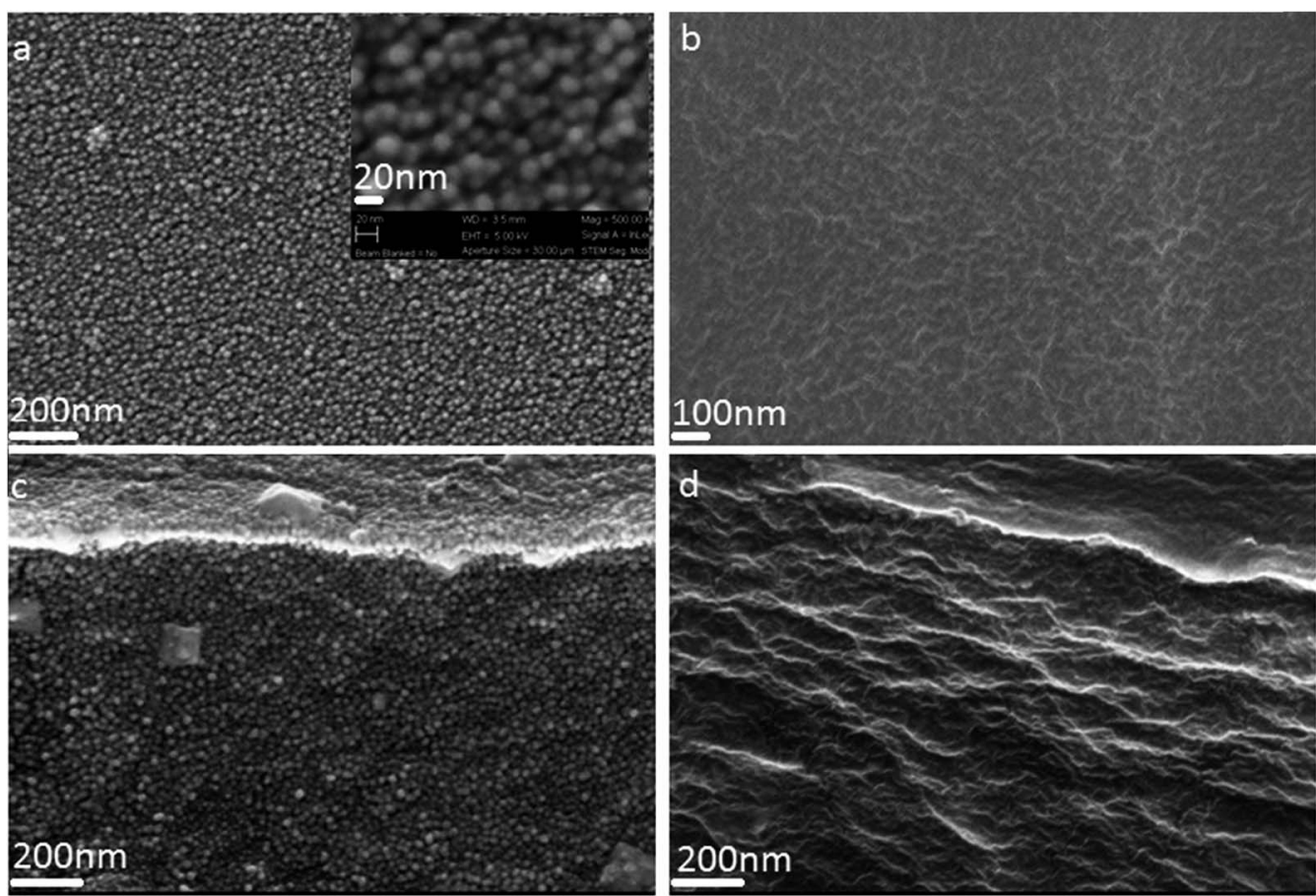

Fig. 11 (Top and cross-section) FESEM views of the UV patterned sample: (a) and (c) UV irradiated part, while (b) and (d) non-irradiated part. The inset to the part (a) shows the top view at higher magnification to present the close distribution and sizes of the Ag NPs. 
layer. In Fig. 9b the C1s HR peaks, collected during depth profile, are also reported. The relative intensities can be considered almost constant during the 160 sputtering cycles. However there is a strong difference between the first HR spectrum (collected before the sputtering procedure) which shows a higher peak at $286.0 \mathrm{eV}$, which is due to $\mathrm{C}-\mathrm{O}$ bond, and a smaller one at $288.3 \mathrm{eV}$ due to $\mathrm{C}=\mathrm{O}$ bond, while after the first sputtering cycle the $\mathrm{C}-(\mathrm{C}, \mathrm{H})$ component at $284.5 \mathrm{eV}$ becomes predominant in the remaining peaks. This is a confirmation of the surface oxidation of polymer network, which was observed previously due to the UV irradiation.

Finally patterning experiments were conducted. A film cured in the visible range was then exposed to UV radiation by using a NXQ 4006 Mask Aligner, provided by Neutronix Quintel. The mask aligner is equipped with a UV broad band, high pressure $\mathrm{Hg}$ vapor lamp. Different structures were patterned with a dose of $9 \mathrm{~J} \mathrm{~cm}^{-2}$ obtaining geometries with a definition down to 10 $\mu \mathrm{m}$ (see Fig. 10 and ESI $\dagger$ ). After the patterning step the films were subjected to a washing step in order to remove unreacted silver salts and stabilize the material.

FESEM images of the patterned and unpatterned area of the samples are reported in Fig. 11. Both the top view and the crosssection images of the patterned area show relatively uniform size and distribution of the NPs, while the unpatterned area does not show any NP.

Successful removal of silver salts is demonstrated also by time stability of the patterned sample. In Fig. S32 $\uparrow$ two pictures of the same sample were taken after 6 months in room conditions (environmental sunlight, humidity, temperature) showing very good stability.

\section{Experimental}

\section{Materials}

Poly(ethyleneglycol) dimethacrylate (PEGDMA, $M_{\mathrm{w}}$ 575, SigmaAldrich) was used as photocurable monomer. Silver nitrate (Sigma-Aldrich) was employed as precursor of Ag NPs.

Two distinct photoactivating systems were used: the first one, active in the visible range, was used for carrying out the polymerization mechanism. It was a Norrish II type photoinitiating system composed by camphorquinone (CQ, SigmaAldrich) and ethyl 4(dimethylamino)benzoate (EMBO, Alfa Aesar) as H-donor. The second one was Irgacure 1173 (BASF), a Norrish I UV-photoactive compound exploited for supporting the silver nitrate photoreduction. Ethanol (SigmaAldrich) was used as solvent and in the washing step. All the chemicals were used as received without any further purification.

\section{Sample preparation}

In a typical formulation, $10 \mathrm{mg}$ of CQ and $10 \mathrm{mg}$ of EMBO were solvated in ethanol $(1 \mathrm{~g})$ and then different amounts of silver nitrate were added to the solution (ranging from $10 \mathrm{mg}$ to 100 $\mathrm{mg}$ ). The obtained solution was then added to $1 \mathrm{~g}$ of PEGDMA. Finally Irgacure 1173 was added ( 2 phr with respect to the amount of monomers).
Typically, the obtained formulation was coated on a glass slide using a wire-wound bar (nominal thickness $200 \mu \mathrm{m}$ ). For measurements that needed particular film thickness, sample preparation will be described later. The photopolymerization process was carried out placing the sample in a home-made device designed for this purpose (see ESI $\dagger$ ) and then irradiating with a visible lamp (Hamamatsu LC8 with visible bulb and a cut-off filter below $400 \mathrm{~nm}$ equipped with $8 \mathrm{~mm}$ light guide) in nitrogen atmosphere. The subsequent silver reduction was performed by UV-exposing the cured samples for different times using a Hamamatsu LC8 with UV bulb equipped with $8 \mathrm{~mm}$ light guide. In both cases light intensity was $50 \mathrm{~mW} \mathrm{~cm}^{-2}$.

The patterning was performed by exposing the already cured film to UV radiation by using a NXQ 4006 Mask Aligner, provided by Neutronix Quintel. The mask aligner is equipped with a UV broadband, high pressure $\mathrm{Hg}$ vapour lamp (light intensity $10 \mathrm{~mW} \mathrm{~cm}^{-2}$ ). Silver salt residuals were washed by soaking the samples for 4 hours in ethanol.

\section{Characterization methods}

Real-time rheological measurements were performed using an Anton Paar rheometer (Physica MCR 302) in parallel plate mode with the Hamamatsu LC8 visible fiber lamp previously described. The lower fixture of the rheometer is a large quartz glass plate, while the top one is a $25 \mathrm{~mm}$ diameter metal plate. The gap between the two was set to $0.2 \mathrm{~mm}$ and the sample was kept at a constant temperature $\left(25^{\circ} \mathrm{C}\right)$ and under constant shear frequency of $1 \mathrm{rad} \mathrm{s}^{-1}$, light was turned on after 3 minutes in order to stabilize the system. Concomitant changes in viscoelastic material moduli during polymerization $\left(G^{\prime}, G^{\prime \prime}\right)$ were measured as a function of exposure time. The measurements were carried out in the linear viscoelastic region (strain amplitude $1 \%$ ).

FT-IR transmittance spectra were collected on a Tensor 27 FTIR Spectrometer (Bruker). The samples were prepared by coating the photocurable formulations on a $\mathrm{Si}$ wafer with a wound wire bar (nominal thickness $25 \mu \mathrm{m}$ ). The averaged signal was collected with a resolution of $2 \mathrm{~cm}^{-1}$ from 4000 to $400 \mathrm{~cm}^{-1}$. IR spectra were collected prior and after 1 min visible irradiation in $\mathrm{N}_{2}$ atmosphere. Another spectrum was collected after 1 min under UV light. Double bonds conversion was determined as decrease of $\mathrm{C}=\mathrm{C}$ peak centered at $1640 \mathrm{~cm}^{-1}$ normalized by the carbonyl peak $\left(1720 \mathrm{~cm}^{-1}\right)$.

The insoluble fraction (gel content) of the cured products was determined according to the standard test method ASTM D2765-84. The samples were held in a metal net, accurately weighted and, subsequently, submitted to extraction for 24 hours at room temperature with $\mathrm{CHCl}_{3}$ to dissolve the not-crosslinked polymer. Then the samples were dried overnight at $60^{\circ} \mathrm{C}$. The insoluble fraction percentage was determined as weight difference before and after solvent extraction.

Differential scanning calorimetry (DSC) experiments were performed using a Netzsch DSC 204 F1 Phoenix instrument, equipped with a low temperature probe. The experiments were carried out between -80 and $40{ }^{\circ} \mathrm{C}$ with a scan rate of $10{ }^{\circ} \mathrm{C}$ $\min ^{-1}$ in nitrogen atmosphere $\left(20 \mathrm{ml} \mathrm{min}^{-1}\right)$. For each sample, 
the same heating module was applied and the final heat flow value recorded during the second heating cycle. The $T_{\mathrm{g}}$ was defined as the midpoint of the heat capacity change observed in the DSC thermogram.

Thermogravimetric analysis (TGA) was performed with a Netzsch TG $209 \mathrm{~F} 1 \mathrm{Libra}$ instrument. All samples were heated between $30{ }^{\circ} \mathrm{C}$ and $800{ }^{\circ} \mathrm{C}$ at a heating rate of $10^{\circ} \mathrm{C} \mathrm{min}{ }^{-1}$ in air flow of $20 \mathrm{ml} \mathrm{min}^{-1}$. For control of the measurements as well as for data acquisition, NETZSCH PROTEUS 32-bit Software was employed with Advanced Software packages like c-DTA (calculated DTA-signal), Super-Res (rate-controlled mass change) and Thermokinetics.

The UV-visible spectra were recorded by means of a double beam Lambda 40 instrument (Perkin-Elmer). The range between 280 and $800 \mathrm{~nm}$ was monitored with a scan step of 1 $\mathrm{nm}$. All the experiments were performed on $100 \mu \mathrm{m}$ films coated on a glass slide.

Optical microscopy images were taken by using a Zeiss Axio A1 Microscope.

Morphologic characterization was performed in top and cross-section views by Field Emission Scanning Electron Microscopy (FESEM, ZEISS Dual Beam Auriga). Cross-section samples were prepared by fracturing them in liquid nitrogen. For these experiments the samples were prepared on a silicon wafer. Prior to FESEM observation samples were coated by a 8 $\mathrm{nm}$ thick Pt film via sputtering for improving electrical conductance.

A PHI 5000 Versaprobe Scanning X-ray Photoelectron Spectrometer (monochromatic Al K- $\alpha$ X-ray source with $1486.6 \mathrm{eV}$ energy, $15 \mathrm{kV}$ voltage and $1 \mathrm{~mA}$ anode current), was used to investigate surface chemical composition. A spot size of $100 \mu \mathrm{m}$ was used in order to collect the photoelectron signal for both the high resolution (HR) and the survey spectra. Different pass energy values were employed: $187.85 \mathrm{eV}$ for survey spectra and $23.5 \mathrm{eV}$ for HR peaks. All samples were analyzed with a combined electron and Ar ion gun neutralizer system in order to reduce the charging effect during the measurements. All corelevel peak energies were referenced to C1s peak at $284.5 \mathrm{eV}$ and the background contribution in HR scans was subtracted by means of a Shirley function. ${ }^{56}$ Spectra were analysed using Multipak 9.6 dedicated software. Depth profile, by means of an $\mathrm{Ar}^{+}$flux at $2 \mathrm{kV}$ accelerating voltage, has been performed in an alternate mode with sputtering cycles of $1 \mathrm{~min}$ each.

\section{Conclusions}

In this work we presented a novel method for obtaining polymeric NCs selectively inhomogeneous from the compositional and functional point of view starting from a single photocurable formulation containing photosensitive metal precursors. A dual step light irradiation process was developed: in the first step, a photopolymerization process was carried out by employing visible radiation while in the second step, a photolithographic technique was exploited in order to generate silver NPs only in UV-exposed areas of the polymeric film. Finally a washing step was carried out in order to extract all the unreduced silver salts and stabilize the material. Great control over NP size distribution is achieved, as well as straightforward transfer of complex geometries, in view of functional electronic/plasmonic/ photonic applications, getting rid of unreacted precursors.

Visible induced polymerization process was investigated as well as UV-induced nucleation and growth of silver NPs in the polymeric matrix. A progressive migration of Ag NPs during UV irradiation was evidenced and studied, a thermally activated phenomenon was proposed as explanation, passing through the formation of $\mathrm{Ag}$ clusters and their migration in the polymer matrix. Finally XPS and TGA measurements confirmed the efficiency of the washing step, thus evidencing the reliability of the proposed approach.

This technique opens new perspectives in the realization of multifunctional materials with possible applications in optics, plasmonics, electronics and sensors. It proved to be fast, easy and possibly up-scalable. Moreover it is envisaged to be further improved by applying region-selective techniques for the generation of metal NPs such laser induced growth/sintering, thus leading to the developing of $3 \mathrm{D}$ structures within the polymeric matrix.

\section{Acknowledgements}

The authors gratefully thank Dr Flaviana Calignano for helping in graphical improvement of the paper.

\section{References}

1 J. C. Eloi, L. Chabanne, G. R. Whittell and I. Manners, Mater. Today, 2008, 11, 28-36.

2 P. Romero-Gómez, F. Pastorelli, P. Mantilla-Pérez, M. Mariano, A. Martínez-Otero, X. Elias, R. Betancur and J. Martorell, J. Photonics Energy, 2015, 5, 057212.

3 S. Yao and Y. Zhu, Adv. Mater., 2015, 27, 1480-1511.

4 C. Hanisch, N. Ni, A. Kulkarni, V. Zaporojtchenko, T. Strunskus and F. Faupel, J. Mater. Sci., 2011, 46, 438445.

5 R. M. Mutiso, J. M. Kikkawa and K. I. Winey, Appl. Phys. Lett., 2013, 103, 223302.

6 S. H. Yeom, B. H. Kang, K. J. Kim and S. W. Kang, Front. Biosci., 2011, 16, 997-1023.

7 L. S. Nair and C. T. Laurencin, J. Biomed. Nanotechnol., 2007, 3, 301-316.

8 H. Palza, Int. J. Mol. Sci., 2015, 16, 2099-2116.

9 S. Kalia, S. Kango, A. Kumar, Y. Haldorai, B. Kumari and R. Kumar, Colloid Polym. Sci., 2014, 292, 2025-2052.

10 M. Bonini, D. Berti and P. Baglioni, Curr. Opin. Colloid Interface Sci., 2013, 18, 459-467.

11 K. Chrissafis and D. Bikiaris, Thermochim. Acta, 2011, 523, 124.

12 G. V. Ramesh, S. Porel and T. P. Radhakrishnan, Chem. Soc. Rev., 2009, 38, 2646-2656.

13 Z. Wu, D. Wu, T. Zhang, L. Jiang, W. Wang and R. Jin, J. Appl. Polym. Sci., 2006, 102, 2218-2225.

14 D. S. Thompson, L. M. Davis, D. W. Thompson and R. E. Southward, ACS Appl. Mater. Interfaces, 2009, 1, 14571466. 
15 R. D. Deshmukh and R. J. Composto, Chem. Mater., 2007, 19, 745-754.

16 S. W. Lee, D. Liang, X. P. A. Gao and R. M. Sankaran, Adv. Funct. Mater., 2011, 21, 2155-2161.

17 L. I. Dan, J. Huang and R. B. Kaner, Acc. Chem. Res., 2009, 42, 135-145.

18 K. Mallick, M. J. Witcomb, A. Dinsmore and M. S. Scurrell, Langmuir, 2005, 21, 7964-7967.

19 A. Barbieri, G. Accorsi and N. Armaroli, Chem. Commun., 2008, 2185-2193.

20 A. Chiolerio, I. Roppolo and M. Sangermano, RSC Adv., 2013, 3, 3446-3452.

21 R. Nazar, S. Ronchetti, I. Roppolo, M. Sangermano and R. M. Bongiovanni, Macromol. Mater. Eng., 2014, 300, 226233.

22 S. Ravaine, G. E. Fanucci, C. T. Seip, J. H. Adair and D. R. Talham, Langmuir, 1998, 14, 708-713.

23 X. Yang and Y. Lu, Mater. Lett., 2005, 59, 2484-2487.

24 M. Sangermano, Y. Yagci and G. Rizza, Macromolecules, 2007, 40, 8827-8829.

25 Y. Yagci, M. Sangermano and G. Rizza, Polymer, 2008, 49, 5195-5198.

26 M. Sangermano, I. Roppolo and M. Messori, in Photocured Materials, The Royal Society of Chemistry, 2015, pp. 121-133.

27 M. Sangermano, I. Roppolo, V. H. A. Camara, C. Dizman, S. Ates, L. Torun and Y. Yagci, Macromol. Mater. Eng., 2011, 296, 820-825.

28 M. Sangermano, A. Chiolerio, G. Marti and P. Martino, Macromol. Mater. Eng., 2013, 298, 607-611.

29 J. R. Nair, V. S. Ijeri, C. Gerbaldi, S. Bodoardo, R. Bongiovanni and N. Penazzi, Org. Electron., 2010, 11, 1802-1808.

30 H. W. Cui, J. T. Jiu, S. Nagao, T. Sugahara, K. Suganuma, H. Uchida and K. A. Schroder, RSC Adv., 2014, 4, 1591415922.

31 A. Matsubayashi, K. Fukunaga and K. Tanaka, J. Mater. Chem., 2012, 22, 15578-15585.

32 A. Matsubayashi, K. Fukunaga, T. Tsuji, K. Ataka and H. Ohsaki, Langmuir, 2011, 27, 733-740.

33 A. S. Korchev, M. J. Bozack, B. L. Slaten and G. Mills, J. Am. Chem. Soc., 2004, 126, 10-11.

34 T. N. Smirnova, L. M. Kokhtych, A. S. Kutsenko, O. V. Sakhno and J. Stumpe, Nanotechnology, 2009, 20, 405301.

35 K. Vora, S. Kang, S. Shukla and E. Mazur, Appl. Phys. Lett., 2012, 100, 063120.

36 F. Stellacci, C. A. Bauer, T. Meyer-Friedrichsen, W. Wenseleers, V. Alain, S. M. Kuebler, S. J. K. Pond,
Y. Zhang, S. R. Marder and J. W. Perry, Adv. Mater., 2002, 14, 194-198.

37 C. M. Huang, C. H. Yeh, L. Chen, D. A. Huang and C. Kuo, ACS Appl. Mater. Interfaces, 2013, 5, 120-127.

38 S. W. Lee and R. M. Sankaran, Mater. Today, 2013, 16, 117122.

39 J. H. Lee, J. P. Singer and E. L. Thomas, Adv. Mater., 2012, 24, 4782-4810.

40 E. Fantino, A. Vitale, M. Quaglio, M. Cocuzza, C. F. Pirri and R. Bongiovanni, Chem. Eng. J., 2015, 267, 65-72.

41 J. P. Fouassier, X. Allonas and D. Burget, Prog. Org. Coat., 2003, 47, 16-36.

42 J. Shao, Y. Huang and Q. Fan, Polym. Chem., 2014, 5, 41954210.

43 A. Chiolerio, A. Virga, P. Pandolfi, P. Martino, P. Rivolo, F. Geobaldo and F. Giorgis, Nanoscale Res. Lett., 2012, 7, 1-7. 44 S. Stassi, V. Cauda, G. Canavese, D. Manfredi, I. Roppolo, P. Martino and A. Chiolerio, in Handbook of Nanomaterials Properties, ed. B. Bhushan, D. Luo, S. R. Schricker, W. Sigmund and S. Zauscher, Springer, Berlin, Heidelberg, 2014, ch. 33, pp. 179-212.

45 A. A. Aimetti, A. J. Machen and K. S. Anseth, Biomaterials, 2009, 30, 6048-6054.

46 J. W. Stansbury, Dent. Mater., 2012, 28, 13-22.

47 B. D. Fairbanks, M. P. Schwartz, C. N. Bowman and K. S. Anseth, Biomaterials, 2009, 30, 6702-6707.

48 D. K. Božanić, S. Dimitrijević-Branković, N. Bibić, A. S. Luyt and V. Djoković, Carbohydr. Polym., 2011, 83, 883-890.

49 S. Chalal, N. Haddadine, N. Bouslah and A. Benaboura, J. Polym. Res., 2012, 19, 1-8.

50 Y. Xia, Y. Xiong, B. Lim and S. E. Skrabalak, Angew. Chem., Int. Ed., 2009, 48, 60-103.

51 A. I. Henry, J. M. Bingham, E. Ringe, L. D. Marks, G. C. Schatz and R. P. Van Duyne, J. Phys. Chem. C, 2011, 115, 9291-9305.

52 P. Mao, J. Chen, R. Xu, G. Xie, Y. Liu, G. Gao and S. Wu, Appl. Phys. A: Mater. Sci. Process., 2014, 117, 1067-1073.

53 A. Chiolerio, L. Vescovo and M. Sangermano, Macromol. Chem. Phys., 2010, 211, 2008-2016.

54 H. S. Shin, H. C. Choi, Y. Jung, S. B. Kim, H. J. Song and H. J. Shin, Chem. Phys. Lett., 2004, 383, 418-422.

55 M. Milošević, M. Radoičić, Z. Šaponjić, T. Nunney, D. Marković, J. Nedeljković and M. Radetić, J. Mater. Sci., 2013, 48, 5447-5455.

56 D. A. Shirley, Phys. Rev. B: Solid State, 1972, 5, 4709-4714. 